\title{
On modeling the dynamic thermal behavior of electrical machines using genetic programming and artificial neural networks.
}

\author{
ZĂVOIANU, A.-C., KITZBERGER, M., BRAMERDORFER, G. and \\ SAMINGER-PLATZ, $S$.
}




\title{
On Modeling the Dynamic Thermal Behavior of Electrical Machines using Genetic Programming and Artificial Neural Networks
}

\author{
Alexandru-Ciprian Zăvoianu ${ }^{1}$, Martin Kitzberger ${ }^{2}$, Gerd Bramerdorfer ${ }^{2}$, \\ Susanne Saminger-Platz ${ }^{3}$ \\ 1 School of Computing Science and Digital Media, Robert Gordon University, \\ Aberdeen, Scotland, UK \\ 2 Institute for Electrical Drives and Power Electronics, \\ Johannes Kepler University Linz, Austria \\ 3 Department of Knowledge-based Mathematical Systems, \\ Johannes Kepler University Linz, Austria
}

\begin{abstract}
We describe initial attempts to model the dynamic thermal behavior of electrical machines by evaluating the ability of linear and non-linear (regression) modeling techniques to replicate the performance of simulations carried out using a lumped parameter thermal network (LPTN) and two different test scenarios. Our focus falls on creating highly accurate simple models that are well-suited for the real-time computational demands of an envisioned symbiotic interaction paradigm. Preliminary results are quite encouraging and highlight the very positive impact of integrating synthetic features based on exponential moving averages.
\end{abstract}

Keywords: data driven modeling, time series, genetic programming, dynamic thermal behavior, electrical machines, lumped parameter thermal networks

\section{Introduction and Motivation}

Given the multitude of challenges imposed by the present trend of automation and data exchange in manufacturing processes (i.e., Industry 4.0), it is feasible that in the near future ever more electrical machines will not only be optimized according to multiple criteria [4] [12, but will also need to operate inside a symbiotic interaction paradigm. This shift from the current state in which electrical machines (as well as other process components) act as slaves of a master control system will require electrical drives and actuators to actively exchange data with their superior system and operating environment and to incorporate some decision-making autonomy that can ensure good long term operational characteristics.

In this newly envisioned operational context, locally-embedded processors like micro-controllers would require: (i) information regarding the current state of the electrical machine, (ii) information regarding environment conditions, (iii) 
the ability to predict power, maintenance and lifetime reserves for upcoming load conditions. In case of the latter, the usage of highly accurate wear and load models - underpinned by linear and especially non-linear regression techniques - has yielded very promising results 3 .

The ability to model the dynamic (real-time) thermal behavior is very important, as the strong interaction between electromagnetic and thermal aspects is known to affect the operational performance of electrical machines: losses are dependent upon temperature and vice versa. According to [13, traditional approaches for performing an accurate thermal analysis of electrical machines fall within two broad categories:

A. Numerical methods - which group techniques based on finite element analysis or computational fluid dynamics. These approaches are quite general with regard to device geometry but very computationally-demanding.

B. Analytical modeling - which is usually instance specific and requires highly specialized domain expertise during model development, but produces models that are very fast to evaluate and could thus be suitable for real-time approaches.

The state-of-the-art approach in analytical modeling aims to construct and correctly parameterize complex lumped parameter thermal networks (LPTNs) [2] that accurately model the main heat-transfer paths of an electrical machine by aggregating several non-linear loss sources (i.e., copper and iron losses).

\section{Research Focus and Approach}

The aim of the present work is to explore if standard data-driven modeling techniques can be applied to predict the dynamic thermal behavior of electrical machines. The main focus falls on obtaining low-complexity models as these:

- can meet the real-time computational demands of micro-controllers;

- can be interpreted more easily by domain experts (i.e., electrical engineers), thus facilitating both domain acceptance and the future development of mixed thermal models.

While the final modeling scenario will consist of time series that aggregate sensor-based temperature measurements collected from electrical drive prototypes, in this incipient study we focus on modeling data streams obtained using simulation programs with integrated circuit emphasis (SPICE) [14. Thus, using the expert-designed LPTN from Figure 1, we simulate two realistic electrical machine operational scenarios.

Although simplistic, this approach enables an initial comparative analysis of the ability of data-driven modeling approaches to replicate LPTN-based results and allows for a better grounding of expectations regarding future sensor-based performance. Furthermore, by simulating the data, we have:

- full control of the input stream - i.e., the time-dependent values of $P_{-} w, P_{-} s$ and $P_{\_} r$, the three power sources from Figure 1; 


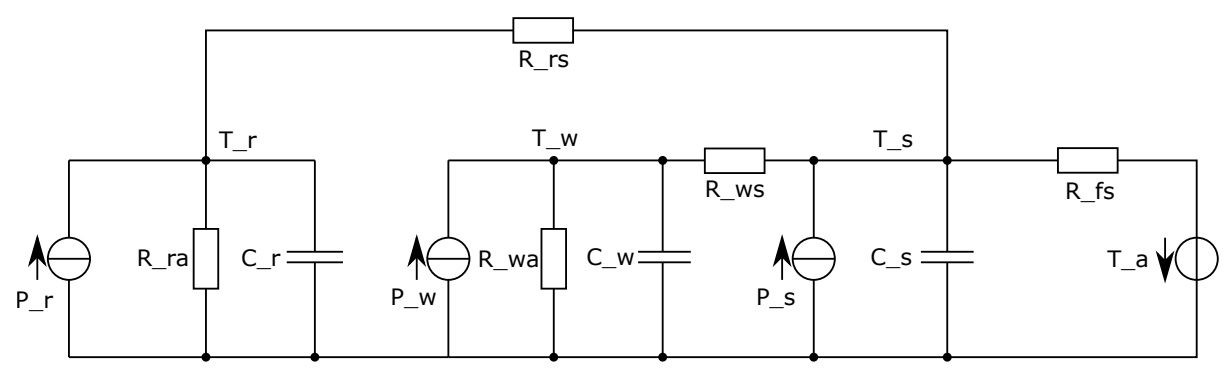

Fig. 1: Basic expert-designed LPTN used for generating data via LTspice ${ }^{\circledR}$.

- cost-effective instant access to the output stream - i.e., (LPTN-estimated) temperatures in the three key parts of the electrical motor we are interested in monitoring: the winding $\left(T \_w\right)$, the stator lamination $\left(T_{-} s\right)$ and the rotor lamination $\left(T \_r\right)$.

Given the characteristics and demands of the envisioned application scenarios (time series data, unknown output values during operation, wish for large prediction horizons, aim for simple models, real-time modeling), we have chosen to model each temperature output at time $t-$ e.g., $T \_w(t)$ - using both the three power inputs at time $t$ - i.e., $P \_w(t), P \_s(t), P \_r(t)$ - and three new synthetic features obtained by computing exponential moving averages (EMAs) for each input. For a given (power) input $\mathbf{x}$, at each generation $t$, we have that:

$$
E M A_{\mathbf{x}}(t)= \begin{cases}\mathbf{x}(t), & \text { if } t=1 \\ 0.01 \cdot \mathbf{x}(t)+0.99 \cdot E M A_{\mathbf{x}}(t-1), & \text { if } t>1\end{cases}
$$

Using the six inputs, we attempted to model each temperature output using three regression techniques: linear regression [9], genetic programming [10] and (shallow) artificial neural networks [7].

\section{Experimental Setup}

\section{The Simulation Scenarios}

Using the LPTN from Figure 1. two operational scenarios were simulated using the LTspice ${ }^{\circledR}$ software. The first simulation lasts for 2000 seconds and concerns a constant load as all three power inputs are simultaneously ramped up to predefined thresholds. The second simulation lasts for 5000 seconds and concerns a more complicated variable load scenario. The input and output time-wise variations for both scenarios are plotted in Figure 2.

\section{Data Sets and Experimental Setup}

While simulation results were initially sampled equidistantly at every 100 milliseconds, the final data sets used for modeling were obtained by keeping only 
Alexandru-Ciprian Zăvoianu et al.
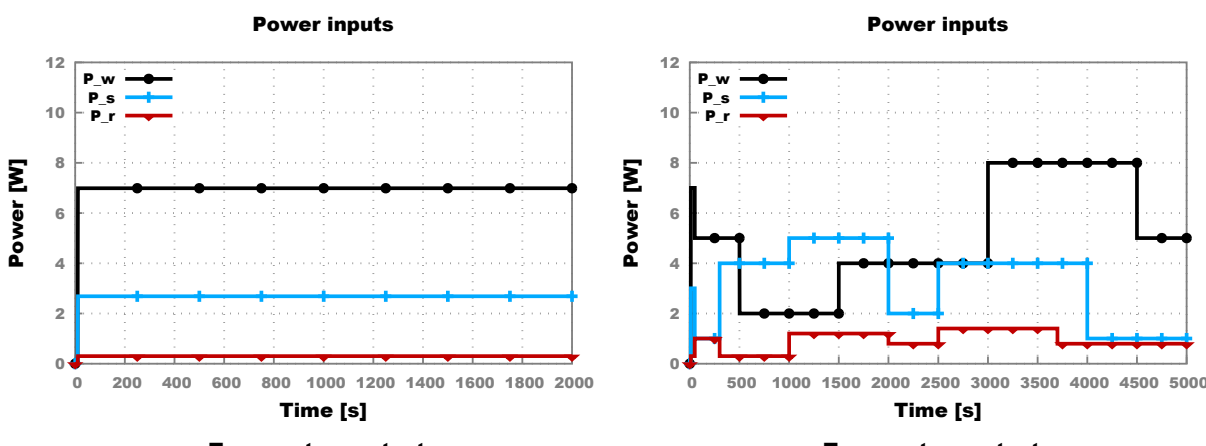

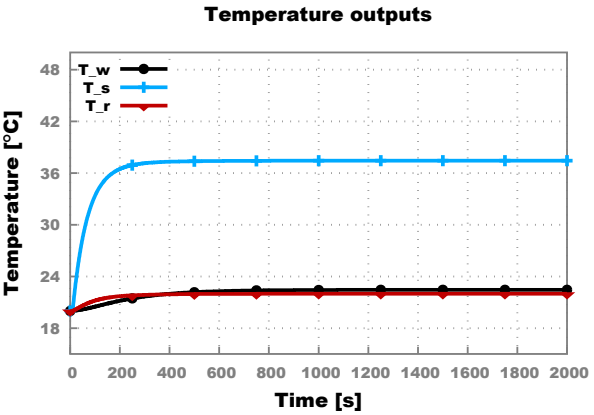

(a) Constant load simulation.

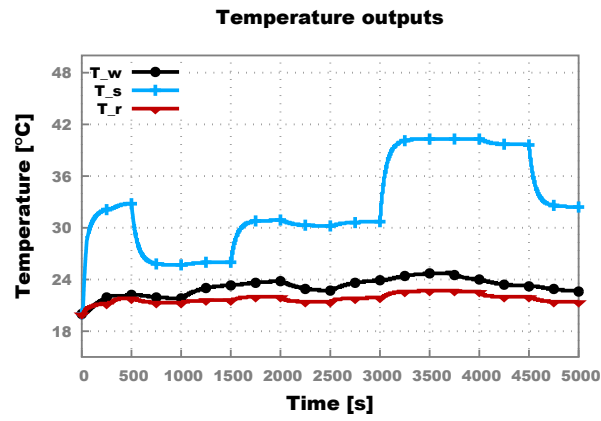

(b) Variable load simulation.

Fig. 2: LPTN-simulated input (powers) and output (temperature) time series.

every $10^{\text {th }}$ sample (subsampling). This resulted in a data set with 2000 samples for the first operational scenario and a data set of 5000 samples for the second one. After computing the associated EMA features for each sample, the data sets were randomly shuffled. Afterwards, during the modeling process, $50 \%$ of the samples were used for training and $50 \%$ for testing.

The plots in Figure 3 show the distribution of training and test samples across the two data sets and are extremely insightful regarding the importance of the synthetic EMA features, as they highlight the contrast between the very low variance of the original power input features (top subplots) and the diverse range of the temperature outputs (bottom subplots).

The linear regression and artificial neural networks (ANN) implementations we tested with are the ones provided by the WEKA machine learning platform $[5]$. In case of the linear modeling, we used a rather standard parameterization: M5 attribute selection [11, elimination of collinear attributes, a value of $10^{-8}$ for the ridge parameter.

For the neural networks, a limited but systematic series of tests using 10fold cross validation yielded that, across both scenarios and all three outputs, one can obtain a simple but highly accurate model with a single hidden layer that contains 6 hidden units (one for each input) by using the following training parameters: a learning rate of 0.2 , a momentum of 0.2 and at most 1000 epochs 

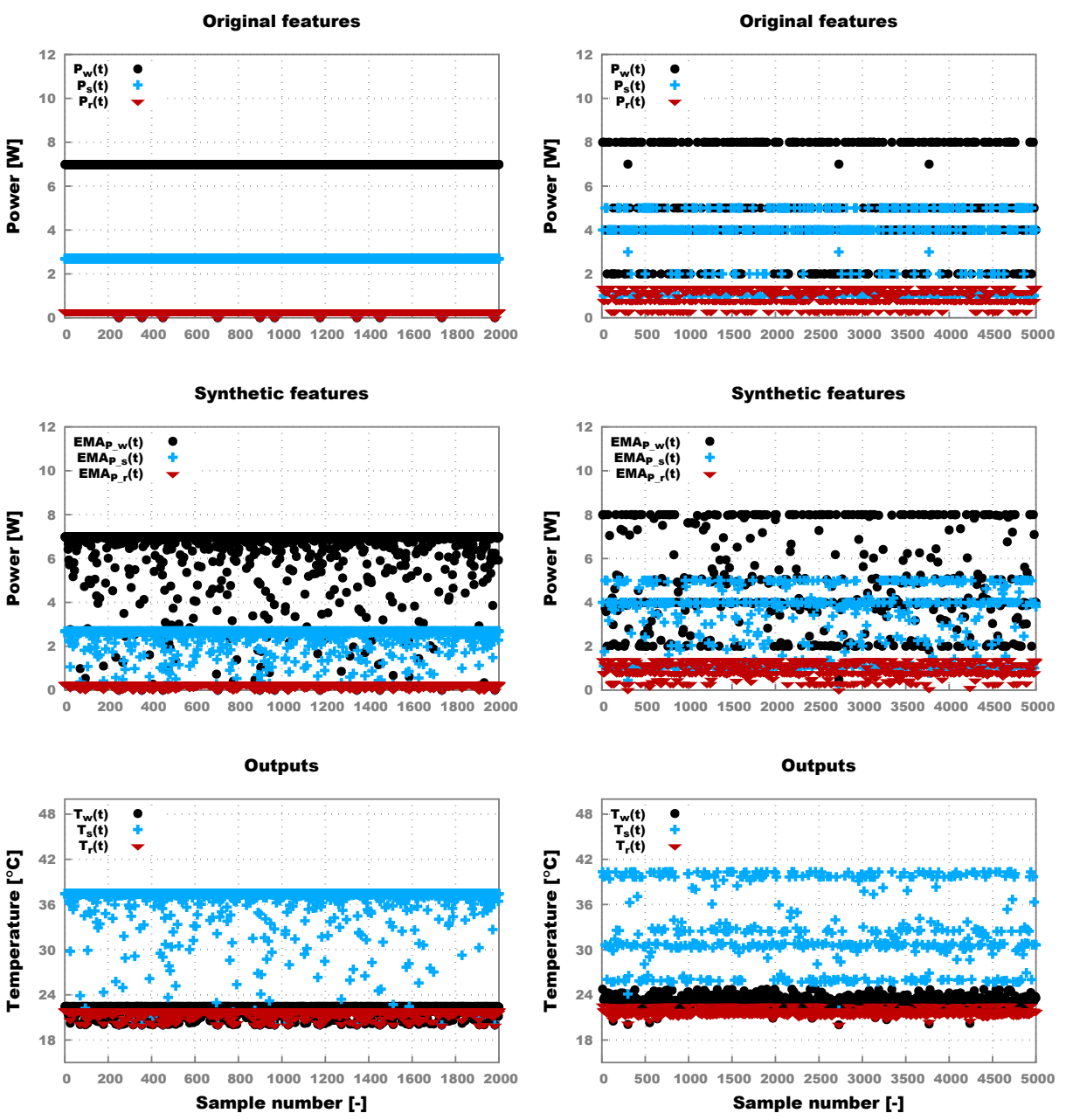

(a) Constant load data set.

(b) Variable load data set.

Fig. 3: Shuffled data sets used for modeling the dynamic thermal behavior.

(i.e., training iterations). As a measure aimed at preventing overfitting, ANN training was stopped before performing all the 1000 iterations whenever the approximation error over a validation set containing $20 \%$ of the initial training samples constantly worsened for 20 consecutive iterations.

The genetic programming (GP) implementation we employed is the one provided by the HeuristicLab framework. More specifically, we opted for an offspring selection flavour of genetic programming (OSGP) that also features constant optimization [8], as this has shown the ability to produce high-quality models in several other application domains [6]. During the run, we evolved 20 generations 
of 500 individuals each. the maximum selection pressure was set to $50 \%$ (i.e., early stopping mechanism) and the best model was chosen based on a validation set containing $20 \%$ of the training samples.

\section{Results - Comparative Performance}

The comparative results of trying to model the two dynamic thermal scenarios using linear and non-linear regression techniques are presented in Table 1 and they indicate that both OSGP and ANNs can deliver very competitive results.

Table 1: Comparative test data performance of regression modeling techniques measured via the coefficient of determination $\left(R^{2}\right)$ and the mean absolute error (MAE). Across both scenarios, the best performance in each temperature - error indicator triple is highlighted.

\begin{tabular}{|l|ccc|ccc|}
\hline \multirow{2}{*}{ Technique-Indicator } & \multicolumn{4}{|c|}{ Constant load scenario } & \multicolumn{3}{|c|}{ Variable load scenario } \\
\cline { 2 - 7 } & $T \_w$ & $T \_s$ & $T \_r$ & $T \_w$ & $T_{\_} s$ & $T_{\_} r$ \\
\hline \hline LinReg- $R^{2}$ & 0.9622 & 0.9980 & 0.8297 & 0.9954 & 0.9978 & 0.9487 \\
ANN- $R^{2}$ & $\mathbf{1 . 0 0 0 0}$ & 0.9990 & 0.9978 & $\mathbf{0 . 9 9 9 8}$ & $\mathbf{0 . 9 9 9 6}$ & $\mathbf{0 . 9 9 9 2}$ \\
OSGP- $R^{2}$ & $\mathbf{1 . 0 0 0 0}$ & $\mathbf{0 . 9 9 9 7}$ & $\mathbf{0 . 9 9 9 9}$ & $\mathbf{0 . 9 9 9 8}$ & 0.9985 & 0.9921 \\
\hline LinReg-MAE & 0.2394 & 0.0108 & 0.1806 & 0.2194 & 0.0156 & 0.1496 \\
ANN-MAE & 0.0117 & 0.0083 & 0.0246 & 0.0489 & $\mathbf{0 . 0 0 7 5}$ & $\mathbf{0 . 0 2 8 0}$ \\
OSGP-MAE & $\mathbf{0 . 0 0 4 1}$ & $\mathbf{0 . 0 0 4 4}$ & $\mathbf{0 . 0 0 4 9}$ & $\mathbf{0 . 0 4 5 5}$ & 0.0125 & 0.0695 \\
\hline
\end{tabular}

It is noteworthy that complementing the two data sets with EMA synthetic features greatly improves prediction accuracy and enables even linear regression models to generally deliver competitive results. Without EMAs, on the simpler constant load scenario, the best regression models can only achieve (test) $R^{2}$ values smaller than 0.55 across all three outputs.

To give some rough insight related to comparative model training times, we mention that constructing the linear regression and ANN models took at most a few seconds when using a single computing core of a standard laptop computer while the OSGP runs took between 20 and 50 minutes when using all four cores.

As there were no attempts to actively limit the size of the evolved GP regression models during the evolutionary run, the resulting solution sizes are bordering the predefined maximal model sizes (i.e., tree depth $=12$, tree length $=50$ ). However, these solutions can be simplified by up to $40 \%$ without any significant loss of accuracy and OSGP has been shown to produce far more 
parsimonious solutions (without sacrificing accuracy) when combined with active runtime bloat control techniques such as iterated tournament pruning and dynamic depth limits [15].

\section{Conclusions and Future Work}

The obtained results show that data-driven techniques can be applied to model (near-perfectly) the dynamic thermal behavior of electrical machines at the level currently obtained by (basic) LPTNs and LTspice ${ }^{\circledR}$ 1. Furthermore, the high accuracy and quite simple structure of the non-linear ANN and OSGP models makes them highly suitable for the envisioned real-time applications. Results indicate a very slight accuracy edge for GP and an overall model simplicity and training time advantage for ANNs.

In the future, we aim to build upon these initial findings and use the datadriven models to construct prediction intervals based on estimated load conditions for different horizons. Furthermore, as sensor data from electrical machine prototypes becomes available, we also plan to replicate the modeling experiment on the real-world data sets. Last but not least, the long term goal is to explore identified synergies and to help the development of hybrid models that combine data-driven and LPTN-based thermal modeling strategies.

\section{Acknowledgments}

This work has been supported by the COMET-K2 "Center for Symbiotic Mechatronics" of the Linz Center of Mechatronics (LCM) funded by the Austrian federal government and the federal state of Upper Austria.

\section{References}

1. https://www. analog.com/en/design-center/design-tools-and-calculators/ ltspice-simulator.html, LTspice - Accessed: 2019-05-30

2. Boglietti, A., Cavagnino, A., Staton, D., Shanel, M., Mueller, M., Mejuto, C.: Evolution and modern approaches for thermal analysis of electrical machines. IEEE Transactions on industrial electronics 56(3), 871-882 (2009)

3. Bramerdorfer, G., Winkler, S.M., Kommenda, M., Weidenholzer, G., Silber, S., Kronberger, G., Affenzeller, M., Amrhein, W.: Using FE calculations and databased system identification techniques to model the nonlinear behavior of PMSMs. IEEE Transactions on Industrial Electronics 61(11), 6454-6462 (11 2014)

4. Bramerdorfer, G., Zăvoianu, A.C.: Surrogate-based multi-objective optimization of electrical machine designs facilitating tolerance analysis. IEEE Transactions on Magnetics 53(8), 1-11 (2017)

5. Eibe, F., Hall, M., Witten, I.: The WEKA Workbench. Online Appendix for Data Mining: Practical Machine Learning Tools and Techniques. Morgan Kaufmann (2016) 
6. Fleck, P., Entner, D., Münzer, C., Kommenda, M., Prante, T., Schwarz, M., Hächl, M., Affenzeller, M.: Box-type boom design using surrogate modeling: Introducing an industrial optimization benchmark. In: Evolutionary and Deterministic Methods for Design Optimization and Control With Applications to Industrial and Societal Problems, pp. 355-370. Springer (2019)

7. Haykin, S.: Neural Networks: A Comprehensive Foundation (2nd Edition). Prentice Hall Inc., Upper Saddle River, New Jersey (1999)

8. Kommenda, M., Kronberger, G., Winkler, S., Affenzeller, M., Wagner, S.: Effects of constant optimization by nonlinear least squares minimization in symbolic regression. In: Proceedings of the 15th annual conference companion on Genetic and evolutionary computation. pp. 1121-1128. ACM (2013)

9. Marquardt, D.W., Snee, R.D.: Ridge regression in practice. The American Statistician 29(1), 3-20 (1975)

10. Poli, R., Langdon, W.B., McPhee, N.F., Koza, J.R.: A field guide to genetic programming. Lulu. com (2008)

11. Quinlan, J.R., et al.: Learning with continuous classes. In: 5th Australian joint conference on artificial intelligence. vol. 92, pp. 343-348. World Scientific (1992)

12. Silber, S., Koppelstätter, W., Weidenholzer, G., Segon, G., Bramerdorfer, G.: Reducing development time of electric machines with SyMSpace. In: 2018 8th International Electric Drives Production Conference (EDPC). pp. 1-5. IEEE (2018)

13. Staton, D., Pickering, S., Lampard, D.: Recent advancement in the thermal design of electric motors. In: Proceedings of SMMA 2001 Fall Technology Conference Emerging Technologies for Electric Motion Industry. pp. 1-11. Durham, North Carolina, USA. (October 3-5 2001)

14. Wang, T.Y., Chen, C.C.P.: SPICE-compatible thermal simulation with lumped circuit modeling for thermal reliability analysis based on modeling order reduction. In: Quality Electronic Design, 2004. Proceedings. 5th International Symposium on. pp. 357-362. IEEE (2004)

15. Zăvoianu, A.C., Kronberger, G., Kommenda, M., Zaharie, D., Affffenzeller, M.: Improving the parsimony of regression models for an enhanced genetic programming process. In: Moreno-Diaz, R., Pichler, F., Quesada-Arencibia, A. (eds.) Computer Aided Systems Theory - EUROCAST 2011, Lecture Notes in Computer Science, vol. 6927, pp. 264-271. Springer Berlin Heidelberg (2011) 